\title{
Occipital neurostimulation in primary headaches: update
}

\author{
A. Proietti Cecchini $\cdot$ M. Leone $\cdot$ V. Tullo \\ M. Curone $\cdot$ P. Di Fiore $\cdot$ G. Bussone
}

(C) Springer-Verlag Italia 2013

\begin{abstract}
A subset of headache patients are chronic and results refractory to standard medical treatments, they are unsatisfied or unable to tolerate the side effects of medications. In the lack of more effective prophylactic treatment, there is need of alternative approach. Migraine is conceptualized as a chronic and potentially progressive disorder. It is conceivable that more aggressive therapeutic efforts could be warranted in drug-refractory chronic migraine. In this prospective, the new, device-based therapies that allow to affect brain function in less invasive ways may represent a therapeutic opportunity. Peripheral occipital neurostimulation resulted in several trials and case reports to be beneficial in a large variety of headache and craniofacial pain disorders, with chronic primary headache the most studied. We comment on our experience in the application of ONS in drug-refractory chronic cluster headache and chronic migraine patients.
\end{abstract}

Keywords Neurostimulation - Occipital nerve · Chronic migraine - Drug refractory - Chronic pain . Cluster headache

\section{Introduction}

Neurosurgical management of chronic pain disorders has been an evolving concept over the past 50 years, still in progress [1].

The lack in the research on new specific drugs in the headache fields has been currently counterbalanced by the

A. Proietti Cecchini $(\bowtie) \cdot$ M. Leone $\cdot$ V. Tullo $\cdot$ M. Curone

P. Di Fiore - G. Bussone

Department of Clinical Neuroscience, Headache Centre,

Neurological Institute IRCCS “C.Besta” Foundation,

Via Celoria, 11, 20133 Milan, Italy

e-mail: proietti.a@istituto-besta.it increasing interest in neuromodulation techniques, with the peripheral neurostimulation approach established as an emerging therapy for refractory chronic headache [2].

Neuroimaging studies prove unequivocally that structural and functional alterations exist in the migrainous brain in between attacks.

Moreover, some of the structural changes, such as white matter lesions load, decreased gray matter density and iron deposition in basal ganglia, correlates with higher attack frequency and longer disease duration, supporting the view of migraine as a progressive disorder [3].

At least some of the morphological changes must be a direct consequence of repeated migraine attacks more than reflect the predisposition to migraine. These abnormalities may probably play a role in the chronification process, as they may account for frequent relapse after transient improvement, i.e., after detoxification treatment, as it involves process either not easily or quickly reversible.

If the risk of structural or homeostatic alterations increases with the migraine load (frequency, severity and disease duration), it is therefore conceivable that more aggressive therapeutic efforts could be warranted in chronic migraine sufferers.

Actually, less invasive techniques may now offer a broader opportunity of treatment for those refractory and/or unsatisfied with pharmacological and conventional therapy.

In this prospective, the new device-based therapies that allow to affect brain function by means of neurostimulation techniques in less invasive ways may represent a therapeutic opportunity.

\section{Occipital neurostimulation}

Occipital nerve stimulation is a technique in which electrodes are placed in the subcutaneous tissue where it 
produces a field of paresthesia within the peripheral distribution of great occipital nerves.

Although the mechanisms of action of peripheral occipital neurostimulation have not been fully elucidated, it results in several trials and case reports to be beneficial in a large variety of headache and craniofacial pain disorders, with chronic primary headache the most studied $[4,5]$.

At least this means that it acts in a non-specific way, even less on the disease generator, as suggested formerly [6].

As a consequence, if CE mark approval in Europe has been obtained-while FDA approval is not yet-it remains evident that peripheral neuromodulation for different chronic headache forms represents up to now a sperimental procedure with off-label indication.

Moreover, occipital neurostimulation (ONS) has already showed in chronic cluster headache patients a beneficial effect in several studies [7-9].

We also moved from hypothalamic deep brain stimulation implants in refractory chronic cluster headache [10] to the peripheral ONS, a safer approach with almost the same rate of improvement. Since 2004, 30 drug-resistant chronic cluster headache patients from our Headache Centre have had implanted an occipital stimulator. $\mathrm{CCH}$ was diagnosed according to the International Headache Society criteria (IHS) and additional criteria to be eligible for neurostimulation were: complete drug-resistance, daily attacks, at least 1 year of the condition and absence of psychiatric disorders. Mean age was 43.3 years, mean illness duration was 15 years (range 9-32) and mean duration of the chronic phase was 7.6 years. Bilateral occipital stimulators were implanted in all patients. The mean follow-up is 46 months (ranges 5-86 months). Eighteen $(60 \%)$ out of the 30 patients showed a headache frequency reduction $>50 \%$ and were defined as responders; in this group, mean headache frequency dropped from 145 attacks per month (5/day) to 13 attacks per month. Twelve patients did not improve but the very first 5 patients of these series implanted in the 2004 and 2005 received occipital stimulation for a too short period, varying from 1 to 5 months. Actually, we were insufficiently experienced and confident in the method. Moreover, it was thought ONS should exert its effect in a short period [6].

Now we know that each patient may have a different time-dependent response to ONS. This means that we must follow-up the ONS treatment on a longer period, up to more than 1 year.

The way to approach drug-refractory chronic migraine has been much more difficult to pursuit due to the extremely heterogeneous population with chronic daily headache. In a very limited case series of four female patients implanted with ONS on an exquisite compassionate base in patients with very low quality of life due to chronic daily headache, we did not obtained any clinical improvement after more than 2-3 years, in spite of the considerable wasting of resource and time allocated, with the evidence of a disappointing imbalance of the cost benefit.

We finally agreed on the importance to select more treatable patients to unravel more reliably the presumed ONS efficacy in migraine. This pushed us to promote the Italian multicenter study named MIGROS [11].

We focused on the selection criteria: primarily we adopted the Manzoni's modified IHS criteria for chronic migraine [12] which limit number of headache-days to maximum 20 per month, excluding those with persistent continuous pain $24 \mathrm{~h}$.

As a consequence, the selected patients are less disabled compared to those with chronic daily headache, but otherwise unsatisfied from the ongoing clinical condition [13]. Those with medication overuse should have passed at least two detoxification treatment without success or with relapse soon after.

We exclude those medication overuse headache patients abusing from ergot, barbiturate, currently very few, or oppioids containing analgesics, in whom detoxification treatment and drug-dependence resolution are mandatory.

Actually in the aim of the 38/2010 law as right to achieve pain relief, in Italy the use of oppioids has increased to one-third, and still it remains long away from the EU average. In expert opinion, there is a general agreement that oppioids are unuseful in primary headache; moreover, carrying the supplementary risk to induce drugdependence and medication overuse headache.

Another main exclusion criterion is the psychopathological comorbidity, which should never reach current clinical relevance in the psychiatric assessment.

\section{Conclusions}

Headache is a biobehavioral disorder resulting from genetic-environmental factors in such a way that headache strictly mirrors the individual biography, rise and falls, in a complex game of emotional, autonomic, neuroendocrine adaptive responses.

In a number of cases, it became chronic, compounded by the presence of psychopathological disorders and maladaptive behavior.

This account for both the great individual suffering and social cost, and why they are often perceived as 'difficult', resistant to treatment, till the final stigma of 'psychiatric'.

Most probably the right dimension is that really the way we cope with emotions has implication in physical pain. It is not a matter of psychogenic pain but rather a dynamic interaction of biological and psychosocial factors, mostly important in the chronification process [14]. To neglect this 
aspect may mean to miss the real nature of the individual suffering and the opportunity to deal with it by a multimodal approach [15].

Paradoxically, this is the point at which the personal experience in neuromodulation techniques in 'difficult' patients has lead: from an exquisite biomedical approach to physical pain to address the more complex psychological component of suffering, often in patients without a defined psychiatric diagnosis according to DSM-IV, both clinical syndromes (Axis I) and/or personality disorders (Axis II).

Formerly, it has been reputed that chronic headache was almost due to medication overuse but then clinical evidence does not support further that simplistic view.

As such, a simple intervention cannot properly manage chronic headache.

A multidisciplinary approach should be in line with a modern biopsychosocial model of chronic pain [16].

It is important for the pharmacological approach as well as for the recent neurostimulation techniques which more properly should be considered as an opportunity to add and enlarge the therapeutic options for those drug-refractory chronic headache patients in a highly disabling condition.

Conflict of interest The authors certify that there is no actual or potential conflict of interest in relation to this article.

\section{References}

1. Reed KL (2013) Peripheral neuromodulation and headaches: history, clinical approach, and considerations on underlying mechanisms. Curr Pain Headache Rep 17(1):305

2. Magis D, Schoenen J (2012) Advances and challenges in neurostimulation for headaches. Lancet Neurol 11(8):708-719

3. Lakhan SE, Avramut M, Tepper SJ (2013) Structural and functional neuroimaging in migraine: insights from 3 decades of research. Headache 53:46-66

4. Jenkins B, Tepper SJ (2011) Neurostimulation for primary headache disorders, part 1: pathophysiology and anatomy, history of neuromodulation in headache treatment, and review of peripheral neuromodulation in primary headaches. Headache 51(8):1254-1266

5. Silberstein SD, Dodick DW, Saper J, Huh B, Slavin KV, Sharan A, Reed K, Narouze S, Mogilner A, Goldstein J, Trentman T, Vaisma J, Ordia J, Weber P, Deer T, Levy R, Diaz RL, Washburn SN, Mekhail N (2012) Safety and efficacy of peripheral nerve stimulation of the occipital nerves for the management of chronic migraine: results from a randomized, multicenter, double-blinded, controlled study. Cephalalgia 32(16):1165-1179

6. Matharu MS, Bartsch T, Ward N, Frackowiak RS, Weiner R, Goadsby PJ (2004) Central neuromodulation in chronic migraine patients with suboccipital stimulators: a PET study. Brain 127(Pt 1): 220-230

7. Burns B, Watkins L, Goadsby PJ (2009) Treatment of intractable chronic cluster headache by occipital nerve stimulation in 14 patients. Neurology 72(4):341-345

8. Magis D, Gerardy PY, Remacle JM, Schoenen J (2011) Sustained effectiveness of occipital nerve stimulation in drug-resistant chronic cluster headache. Headache 51(8):1191-1201

9. Fontaine D, Christophe Sol J, Raoul S et al (2011) Treatment of refractory chronic cluster headache by chronic occipital nerve stimulation. Cephalalgia 31(10):1101-1105

10. Leone M, Franzini A, Proietti Cecchini A, Bussone G (2013) Success, failure, and putative mechanisms in hypothalamic stimulation for drug-resistant chronic cluster headache. Pain 154(1):89-94

11. Proietti Cecchini A, Leone M, Manzoni GC, Torelli P, Bussone G (2012) Drug-resistant chronic migraine: the Italian GON project. Neurol Sci 33(Suppl 1):S33-S35

12. Manzoni GC, Bonavita V, Bussone G, Cortelli P, Narbone MC, Cevoli S, D'Amico D, De Simone R, Torelli P, ANIRCEF (Associazione Neurologica Italiana Ricerca Cefalee) (2011) Chronic migraine classification: current knowledge and future perspectives. J Headache Pain 12(6):585-592

13. Manzoni GC, Grisendi I, Torelli P (2011) ICHD-3: what changes do we need regarding migraine? Curr Pain Headache Rep 15(3):170-176

14. Apkarian AV (2011) The brain in chronic pain: clinical implications. Pain Manag 1(6):577-586

15. Bussone G, Grazzi L, Panerai AE (2012) Pain, emotion, headache. Headache 52(2):98-101

16. Rief W, Kaasa S, Jensen R, Perrot S, Vlaeyen JW, Treede RD, Vissers KC (2012) New proposals for the International Classification of Diseases-11 revision of pain diagnoses. J Pain 13(4): $305-316$ 